\title{
Mendelian randomization: loosening the Gordian knot of testosterone and male ageing
}

\author{
David J Handelsman \\ Asian Journal of Andrology (2013) 15, 579-581; doi:10.1038/aja.2013.71; published online 17 June 2013
}

\begin{abstract}
T is unknown whether the reduced blood testosterone among unselected older men ("andropause") compared to healthy younger men is due to ageing per se or as a non-specific adaptive reaction to chronic diseases accumulating during ageing. A new data analytical technique, Mendelian Randomisation, sheds light on this issue by suggesting that male ageing does not significantly cause lowering of blood testosterone. This shifts the focus from whether treatment of andropause is justified to whether testosterone is safe and effective treatment for the major co-morbidities of ageing such as cardiovascular disease, obesity and chronic organ failure syndromes.

The well-known aphorism, attributed variously to Newton or older philosophers, that to see further than others we must stand on the shoulders of giants, deflects credit for the extraordinary advances of post-Enlightenment science to instrumental tools rather than unique personal vision. Progress has depended on developing and transmitting conceptual and methodological tools which, when coupled with human's probably fixed intelligence, create the remarkable modern edifice of science and technology. Like road maps, from a suitable distance progress in science may seem smooth, but up close it is comprised of important quantal steps, jumps arising from new ideas or tools. If the advances are big enough, they are called paradigm shifts but the more frequent, smaller but definite advances (the 'breakthroughs' that infest media 'science'), are the daily fare of working scientists. In contemporary medical science, introducing new or even borrowed tools often refreshes an area where progress has reached a plateau.
\end{abstract}

ANZAC Research Institute, University of Sydney, Sydney, NSW 2139, Australia Correspondence: Professor DJ Handelsman (djh@ anzac.edu.au)
In that context, the role of testosterone in male ageing is a contentious, intensely studied area as reflected in four recent excellent but inconclusive meta-analyses. ${ }^{1-4}$ This represents a plateau clearly needing a disruptive new concept or tool to facilitate deeper understanding. Since the first studies of male reproductive ageing, made feasible by the invention of testosterone immunoassays in the early 1970s, it has been recognized that older men typically have lower circulating testosterone concentrations. Over the subsequent four decades, the design of numerous studies has sharpened progressively with better definition of study population (from convenience to well-defined samples), increasing sample size (from dozens to thousands), establishing longitudinal cohorts (rather than single snapshot cross-sectional studies), superior mass spectrometry steroid assays (rather than immunoassays) and data analysis methods for cohort studies subject to confounding. Nevertheless, all these studies have remained observational and thereby lacking the truly great twentieth century innovations in clinical research, randomisation and the placebo control, the sine qua non for definitive research findings. As a result, the interpretation of findings from the observational studies of ageing remains as much a creative and imaginative art as a rigorous science, compared with decisive experimental studies. Yet the prospect of a randomized, placebo-controlled of testosterone replacement therapy in male ageing, which the 2004 Institute of Medicine report ${ }^{5}$ concluded was not then warranted by the available preliminary evidence, appears to be both remote and receding.

A fundamental consideration in interpreting the clinical significance of the declining blood testosterone concentrations associated with male ageing is whether this association, too consistent for pure coincidence, is cause or effect. According to the Andropause hypothesis, male ageing is an androgen deficiency state whereby reduced blood testosterone contributes to the detrimental age-related somatic effects which may therefore be rectifiable by physiological testosterone replacement therapy. This draws on the therapeutic metaphor of unequivocal hypogonadism such as young men with pathological, organic hypogonadism or post-menopausal women in whom hormone deficiency states are rectifiable by physiological hormone replacement, subject to the safety of specific regimens. Conversely, the association may be an adaptive response of a functional hypothalamic-pituitary-testicular axis to adverse environmental circumstances (e.g., the accumulating comorbidities of ageing) causing a nonspecific, modest reduction in blood testosterone. In the latter case, blood testosterone concentration is a surrogate metric, a nonspecific biomarker, for ill-health and no justification for physiological testosterone therapy. The plethora of clinical research studies on testosterone and male ageing that continue to appear hover indecisively over that fence.

An interesting break in this stasis is represented by the paper by Haring et al. ${ }^{6}$ which makes an important contribution by introducing a new analytical tool, Mendelian randomisation (MR). ${ }^{7}$ First described 20 years ago $^{8}$ as a means to overcome the perennial problem of confounding in observational studies, Haring et al. adapt this MR approach to the question of whether the regular association of lowered blood testosterone levels with cardiovascular disease/events (including death) in observational epidemiology is a cause or a consequence of the disease. A key distinction between a cause and an effect is the temporal sequence whereby a cause must precede any effect. The dilemma of confounding occurs because, in theory, any variable which could be considered hypothetically a cause, but which may occur before or after an event of interest, could instead be an effect. A single 
snap-shot, cross-sectional study cannot distinguish between those two possibilities. However, genetic factors fixed at conception eliminate the possibility of reverse causation because no environmental effect could cause the genotype. Thus, coupling the random allocation of genetic alleles at conception with the unidirectionality of time uniquely can disrupt the otherwise intractable confounding of cause and effect in observational epidemiology.

MR implements this insight by parsing the confounding of blood testosterone, with its susceptibility to environmental effects, as a predictor of cardiovascular disease using intervening variables, ${ }^{9}$ typically genotypes randomly allocated and fixed at conception but which predict the confounded variable of interest. In this case, the intervening variable is SHBG whereby the random genetic allocation of SHBG alleles at conception creates functional polymorphisms that determine differing blood testosterone concentrations. By this means, the unconfounded genotype (SHBG polymorphisms) is substituted for the confounded predictor (i.e., blood testosterone) in the modelling. Thus, using intervening genetic variables reorients the problem of confounding from a handicap into a tractable genetic tool. Using this ingenious methodological switch, the authors utilize two genetic polymorphisms of the SHBG gene to show that, although confirming the numerous previous observations that blood testosterone was consistently associated with all-cause mortality, these associations were consistently non-causal when analysed by the MR approach utilizing SHBG genotype as the intervening variable. These are important and enlightening new findings which warrant careful consideration for their implications as well as for their assumptions.

A key assumption of MR is that the relationship of the genetic marker (in this case, SHBG polymorphism) to outcome involves only a singular biological mechanism. In this case, this assumes that the outcome is only influenced by SHBG acting through its effects on the confounded variable, blood testosterone concentrations. However, although SHBG has other effects on testosterone transport, uptake and action, it is a further assumption that those are full reflected by SHBG effects on blood testosterone concentrations. For example, SHBG influences metabolic clearance rate, ${ }^{10}$ tissue uptake of blood testosterone at least partly via uptake of SHBG-bound testosterone complexes ${ }^{11-13}$ and that a SHBG polymorphism can change the SHBG binding affinity for testosterone ${ }^{14}$ violating the assumptions of the widely used but erroneous calculations of 'free' testosterone. ${ }^{15-17}$ Polymorphic genetic effects on these mechanisms could undermine the validity of MR.

Another assumption is that genetic effects are fixed from conception whereas epigenetic effects violate this assumption. In this case, however, there is no evidence or reason to believe that epigenetic effects influence the genotype or phenotype of interest in this study; nevertheless, as usual, the absence of evidence is not evidence of the absence.

A potential limitation of negative MR model findings is insufficient power if the polymorphic genetic SHBG effect on the counfounded predictor variable (blood testosterone) is too small relative to the dispersion of that variable in the study population when considering the outcome of interest (all-cause mortality). In this study, the magnitude of the effects of the intervening variable (SHBG polymorphism) on blood testosterone is comparable with the effects of male ageing so this assumption appears sound. On the other hand, the limited power of RM models ${ }^{18}$ themselves require large sample size (where the number of outcomes rather than the study population as a whole counts) and in this case, appeared to preclude extending the analysis to cardiovascular mortality from the study, an specific outcome of even greater interest. ${ }^{19}$

The elegant new perspective provided by Haring et al.'s MR analysis warrants thoughtful integration into the patchwork of knowledge about androgens and male ageing. While reinforcing recent meta-analyses that consistently confirm blood testosterone is a useful but nonspecific biomarker for survival, $^{1-4}$ it provides an important counterpoint to the simplistic belief that the mere lowering of blood testosterone constitutes a treatable androgen deficiency state which may rectify somatic aspects of male ageing. That naive belief, encouraged by permissive clinical guidelines, ${ }^{20,21}$ has led to the present epidemic of testosterone prescribing. ${ }^{22}$ On the contrary, it supports growing evidence that age per se is not associated with any lowering of blood testosterone whereas the comorbidities that accumulate with ageing are. ${ }^{23}$ Haring et al. also provides valuable balance to a recent retrospective study in older US veterans showing that testosterone-treated men survived longer than those not testosterone treated, ${ }^{24}$ an uncontrolled observation easily misinterpreted as testosterone treatment prolongs survival in older men. As outlined in the excellent accompanying editorial, ${ }^{25}$ this finding reflects the bias of a nonrandomized study whereby testosterone was selectively prescribed to men with better prognosis by doctors judging they could benefit from hoped-for improved quality of life. In the end, however, extracting causal relationships from non-experimental, observational data, whether in the form of cohort studies or even MR re-analysis, can never be the alchemical magic one might wish for. But this inventive attempt to do so by Haring et al. does extract at least an intriguing and promising new insight.

1 Corona G, Rastrelli G, Monami M, Guay A, Buvat J et al. Hypogonadism as a risk factor for cardiovascular mortality in men: a meta-analytic study. Eur J Endocrinol 2011; 165: 687-701.

2 Ruige JB, Mahmoud AM, de Bacquer D, Kaufman JM. Endogenous testosterone and cardiovascular disease in healthy men: a meta-analysis. Heart 2011; 97: 870-5.

3 Araujo AB, Dixon JM, Suarez EA, Murad MH, Guey LT et al. Clinical review: endogenous testosterone and mortality in men: a systematic review and metaanalysis. J Clin Endocrinol Metab 2011; 96: 300719.

4 Fernandez-Balsells MM, Murad MH, Lane M, Lampropulos JF, Albuquerque F et al. Clinical review 1: adverse effects of testosterone therapy in adult men: a systematic review and meta-analysis. J Clin Endocrinol Metab 2010; 95: 2560-75.

5 Liverman CT, Blazer DG. Testosterone and Aging: Clinical Research Directions. In: Board on Health Sciences Policy. Washington, DC: Institute of Medicine: The National Academies Press; 2004. p217.

6 Haring R, Teumer A, Volker U, Dorr M, Nauck M et al. Mendelian randomization suggests non-causal associations of testosterone with cardiometabolic risk factors and mortality. Andrology 2013; 1: 17-23.

7 Smith GD, Ebrahim S. 'Mendelian randomization': can genetic epidemiology contribute to understanding environmental determinants of disease? Int J Epidemiol 2003; 32: 1-22.

8 Gray R, Wheatley K. How to avoid bias when comparing bone marrow transplantation with chemotherapy. Bone Marrow Transplant 1991; 7 (Suppl 3): 9-12.

9 Smith GD, Timpson N, Ebrahim S. Strengthening causal inference in cardiovascular epidemiology through Mendelian randomization. Ann Med 2008; 40: 524-41

10 Petra P, Stanczyk FZ, Namkung PC, Fritz MA, Novy $M L$. Direct effect of sex-steroid binding protein (SBP) of plasma on the metabolic clearance rate of testosterone in the rhesus macaque. J Steroid Biochem Mol Biol 1985; 22: 739-46.

11 Hammes A, Andreassen TK, Spoelgen R, Raila J, Hubner $\mathrm{N}$ et al. Role of endocytosis in cellular uptake of sex steroids. Cell 2005; 122: 751-62.

12 Rosner W, Hryb DJ, Khan MS, Nakhla AM, Romas NA. Sex hormone-binding globulin mediates steroid hormone signal transduction at the plasma membrane. J Steroid Biochem Mol Biol 1999; 69: 481-5.

13 Rosner W, Hryb DJ, Khan MS, Nakhla AM, Romas NA. Androgen and estrogen signaling at the cell membrane via G-proteins and cyclic adenosine monophosphate. Steroids 1999; 64: 100-6. 
14 Ohlsson C, Wallaschofski H, Lunetta KL, Stolk L, Perry JR et al. Genetic determinants of serum testosterone concentrations in men. PLOS Genet 2011; 7: e1002313.

15 Hackbarth JS, Hoyne JB, Grebe SK, Singh RJ. Accuracy of calculated free testosterone differs between equations and depends on gender and SHBG concentration. Steroids 2011; 76: 48-55.

16 Ly LP, Sartorius G, Hull L, Leung A, Swerdloff RS et al. Accuracy of calculated free testosterone formulae in men. Clin Endocrinol (Oxf) 2010; 73: 382-8.

17 Sartorius G, Ly LP, Sikaris K, McLachlan R, Handelsman DJ. Predictive accuracy and sources of variability in calculated free testosterone estimates. Ann Clin Biochem 2009; 46: 13743.
18 Pierce BL, Ahsan H, Vanderweele TJ. Power and instrument strength requirements for Mendelian randomization studies using multiple genetic variants. Int J Epidemiol 2011; 40: 740-52.

19 Haring R, Wallaschofski H, Nauck M, Felix SB, Schmidt CO et al. Total and cardiovascular disease mortality predicted by metabolic syndrome is inferior relative to its components. Exp Clin Endocrinol Diabetes 2010; 118: 685-91.

20 Wang C, Nieschlag E, Swerdloff R, Behre HM, Hellstrom WJ et al. Investigation, treatment, and monitoring of late-onset hypogonadism in males: ISA, ISSAM, EAU, EAA, and ASA recommendations. J. Androl 2009; 30: 1-9.

21 Bhasin S, Cunningham GR, Hayes FJ, Matsumoto AM, Snyder PJ et al. Testosterone therapy in men with androgen deficiency syndromes: an Endocrine
Society clinical practice guideline. J Clin Endocrinol Metab. 2010; 95: 2536-59.

22 Handelsman DJ. Pharmacoepidemiology of testosterone prescribing in Australia, 1992-2010. Med J Aust 2012; 196: 642-5.

23 Sartorius G, Spasevska S, Idan A, Turner L, Forbes E et al. Serum testosterone, dihydrotestosterone and estradiol concentrations in older men self-reporting very good health: the healthy man study. Clin Endocrinol (Oxf) 2012; 77: 755-63.

24 Shores MM, Smith NL, Forsberg CW, Anawalt BD, Matsumoto AM. Testosterone treatment and mortality in men with low testosterone levels. J Clin Endocrinol Metab 2012; 97: 2050-8.

25 Wu FC. Caveat emptor: does testosterone treatment reduce mortality in men? J Clin Endocrinol Metab 2012; 97: 1884-6. 